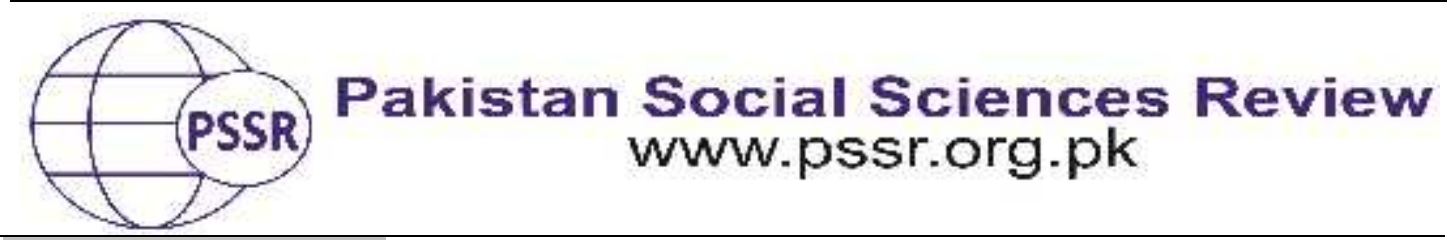

RESEARCH PAPER

\title{
Vulnerability to Emotional Problems among the Parents of the Children with life-threatening Illness and Developmental Disabilities
}

\author{
Muhammad Luqman Khan ${ }^{1}$ Arooj Sarfraz² Rabia Afzal ${ }^{3}$
}

1. Ph. D Scholar, Islamic International University, Islamabad, Pakistan

2. Research Scholar, COMSATS institute of information technology, Islamabad, Pakistan

3. Lecturer, Comwave Institute of Sciences and Information Technology, Islamabad, Pakistan

\section{PAPER INFO}

Received: February 18, 2019

Accepted:

June 24, 2019

Online:

June 30, 2019

Keywords:

Emotional

Problems, Life-

Threatening,

Developmental

Disabilities,

Vulnerability

\section{Corresponding}

Author:

m.luqman.khan.i

iu@gmail.com

\section{ABSTRACT}

The current study is related to about the emotional problems of the parents whose children have life threatening disease and developmental disabilities in Pakistan. The sample included 100 parents as research participants $(\mathrm{N}=100), 50$ were parents $(\mathrm{n}=50)$ of the children with life-threatening illnesses in which 25 were male parents and 25 were female parents. Similarly 50 parents $(n=50)$ of the children with developmental disabilities were taken in which 25 were male parents and other 25 were female parents. The participants were selected from both government hospitals and private clinics and from the institution for the children with special needs. $T$ test has been used for the statistical analysis. The results have shown the significance difference in the levels of emotional problems among parents of children who have life-threatening illnesses and developmental disabilities. On the basis of current results, it is recommended that in future studies gender difference may also be compared.

\section{Introduction}

Parents of the children diagnosed with life-threatening illness or with any developmental disability have to undergo many psychosocial, physical and financial problems. Some problems are of minor level like worthlessness, hopelessness, blue mood, fatigue, cold, dizziness, palpitation, choking, disturbance in sleep and appetite etc, and some can become serious which can be diagnosed as disorders like anxiety disorder and depression. Since there is a unique bond between parents and children and whenever something bad happens to the child, it ultimately affects the parents emotionally and physically. Consequently, if the only child gets sick with some life-threatening illness or have some developmental disability, it could become disastrous for the parents emotionally. The objective of the current research is to examine the difference in vulnerability to emotional problems like stress, anxiety 
and depression in the parents of the children who have any life threatening illness and developmental disabilities.

As becoming a parent is a matter of pride and honor for a person, because it completes one's life and gives the feeling of fulfillment, so parents usually associate their dreams and hopes with their child. But if the child gets a life-threatening illness or seme developmental disability, then the dreams and hopes of the parents that they have associated with their child are shattered. When parents come to know that their child has got some life-threatening illness which can cause death or he/she has some developmental disability which is not curable and it will remain there with him/her throughout his/her life,they develop many emotional, psycho-social and physical problems.

Lot of work has been done in developed countries previously on the relationship of emotional problems in the parents of the children with the lifethreatening illness or any developmental disability, but there are necessary factors to deexplore in the developing countries like Pakistan. The results of the developed countries could not be generalized in Pakistan as the economic, religious, social and educational conditions are different than that of underdeveloped countries. Hence, this study will be a contribution to know about the emotional problems of the parents whose children have life threatening disease and developmental disabilities in Pakistan.

\section{Literature Review}

Steele \& Davies (2006) conducted a study to assess the effects on the parents if their children have any life threatening disease or illness. In the current study, data have been collected from eight families ( 29 members) with the help of observation and interviews. It has been observed that the effects on the parents were pervasive and multidimensional as they had to face emotional, physical economical and spiritual effects in their life as well. This type of stress is closely related to severity level and disability of their children diagnosis and other problem relating to behavior (Vitaliano, Zhang \&Scanlan 2003). If there is high level of autism symptomology, it would increase the parental stress (Hastings \& Johnson, 2001). It has been observed that high level of child's symptoms would increase the stress in the parents (Dunn, Burbine, Bowers \&Tantleff-Dunn, 2001). Children who have intellectual disabilities, the level of stress and anxiety increases in their parents (Dunn et al., 2001; Hastings et al., 2005; Yirmiya \& Shaked, 2005).

Anxiety is defined as an autonomic arousal; the muscles of skeleton affect the situational anxiety and other subjective experience of this effect of anxiety (Lovibond \& Lovibond, 1995). Feldman (1993) has defined the anxiety as feeling of fear and tension and is related to perceptions of uncertainty and threats.

Depression is observed as the state of emotions affected by great sadness, worthlessness and guilt and results in loss of sleep, appetite and agitation. Daoud, 
Dooley \& Gordon (2004) investigated the level of depression in the parents of the children having Duchenne muscular disease. They have observed the level of stress, depression and self-esteem in the care takers of males group who have Duchenne muscular dystrophy disease in a control group. For the data collection, the questionnaire has been based on National Population Health Survey from Statistics Canada and was conducted on the phone calls with 42 parents. The demographic characteristics like province, age, children and marital status of the individuals have been observed. It has been examined that parents of children who have Duchenne muscular dystrophy have high probability of having major depression and had less self-esteem and scores of mastery in comparison to control group.

Frank, Verhulst, Willem, Helbing, Bogers, Domburg\& Elisabeth (2007) conducted a study to examine psychological stress level in parents both mothers and fathers who had any invasive treatment for congenital cardiac disease at least seven years and six months ago. The general health questionnaire and the Utrecht coping list were filled by the parents of children who have four different types of cardiac diagnosis. If there is comparison with the reference groups, it has been observed that parents of children who got treatment for congenital cardiac disease have less distress level, anxiety, less sleep and depression. Mothers have significantly more somatic and affected in comparison to fathers. It has also been observed that families face less disruption and increased closeness while some families have more depression and crisis (Cadman, Rosenbaum, Boyle, \& Oxford, 1991; Cohen, 1999; Steinglass, 1998; Tiedje\& Darling-Fisher, 1996).

Stress may be defined as chronic and non-specific arousal in feelings. It is related to nervous arousal, relaxing problem, agitated, over reactive and also impatient feelings (Lovibond \&Lovibond, 1995). Life threatening illness is known as condition of having risk of premature death because of severe illness but there could be chance of survival into adulthood(Benini, Spizzichino, Trapanotto\& Ferrante, 2008). If there is comparison of mothers and fathers, mothers are more likely to look after the children in their illness and also quit their jobs if required. They have also high level of stress and depression as compared to fathers (Olsson \& Hwang, 2001).

On the basis of literature, followings are the hypotheses of study

H1 There would be a significant difference in the depression levels in the parents of the children with life-threatening illnesses and the parents of the children with developmental disabilities.

H2 There would be a significant difference in the anxiety levels among the parents of the children with life-threatening illnesses and the parents of the children with developmental disabilities.

H3 There would be a significant difference in the stress levels among the parents of the children with life-threatening illnesses and the parents of the children with developmental disabilities. 


\section{Materials and Methods}

The sample included 100 parents as research participants $(\mathrm{N}=100), 50$ were parents $(n=50)$ of the children with life-threatening illnesses in which 25 were male parents and 25 were female parents. Similarly 50 parents $(n=50)$ of the children with developmental disabilities were taken in which 25 were male parents and other 25 were female parents. The participants were selected from both government hospitals and private clinics and from the institution for the children with special needs. The respondents age was between 20 to 50 years and they were based on the criteria like the parents were selected whose children's age were from age since birth to 18 years. Secondly, mothers and fathers of children having life threatening disease and developmental disabilities.

\section{Variables}

\section{Emotional Problems}

Emotional problems here in this study are operationally defined as score obtained from Depression Anxiety Stress Scale (DASS by Aslam and Tariq (2007).

\section{Life-threatening Illness}

Life-threatening illness refers to as such disease which may cause death sooner or later.

\section{Developmental Disability}

Developmental disability is operationally defined as any disability related to cognitive, emotional and physical impairment that appears during birth, infancy or childhood and cause delay or failure to progress through the normal developmental stages of childhood.

\section{Measures}

Translated Urdu version of the Depression, Anxiety and Stress scale (DASS) by Aslam and Tariq (2007) were used to find out depression, anxiety and stress levels in the parents of the children with life-threatening illness. DASS has 42 items, used to measure depression, stress and anxiety. This scale was developed by Lovibond and Lovibond (1995). The Cronbach's alpha values were 0.94, 0.93 and 0.88 for depression, stress and anxiety respectively. The alpha values for the 14- item scales in the normative sample are: $0.91,0.84$ and 0.93 for depression anxiety and stress respectively. DASS contains three sub scales which have 14 items. This instrument is used to measure the dysphoria, hopelessness and life devaluation, anhedonia and inertia. The score ranges from (0-3). In this scale 0 is for do not apply to me at all, 1 is for applies to me to some degree, 2 is for applies to me a 
considerable degree or good part of time, 3 is for applies to me very much. All items are scored positively. The cut off scores for levels of depression are: Mild (10-13), Moderate (14-20), Severe (21-27) and for Extremely Severe (28 and above). For levels of anxiety are: Mild (8-9), moderate (10-14), severe (15-19) and extremely severe (20 and above). And for levels of stress are: Mild (15-18), Moderate (19-25), Severe (2633) and for Extremely Severe (34 and above).

\section{Procedure}

100 parents were selected as the research participants in which 25 were male parents and 25 were female parents of the children with life-threatening illness, similarly 25 male parents and 25 were the female parents of the children with developmental disabilities were included in the sample. Parents of the children with life-threatening illness were taken from Allied Hospital Faisalabad, Al-Haj Siddique Aslam Sheikh Hospital Faisalabad whose children were admitted there; whereas the parents of the children with developmental disabilities were taken from TanzeemUl-Lisan Faisalabad. Depression Anxiety Stress Scale (Aslam \& Tariq, 2007) was administered to check the level of depression, anxiety and stress. Permission letter from head of the department was given to avoid any difficulty in data collection. It was clearly mentioned on the questionnaire that the information of the respondents would be kept safe and used only for the research purpose. T-test has been used for the statistical analysis.

\section{Results and Discussion}

The present study was conducted to find out the emotional problems among the parents of the children with life-threatening illnesses and developmental disabilities. A sample of 100 parents was selected from Allied Hospital Faislabad, AlHaj Siddique Aslam Sheikh Hospital Faisalabad and Tanzeem-Ul-Lisan Faisalabad. Out of those 100 parents, 50 parents $(\mathrm{N}=50)$ including 25 mothers and 25 fathers, were of the children having life-threatening illnesses and rest of $50(n=50)$ parents including 25 mothers and 25 fathers, were the parents of the children having developmental disabilities. Emotional problems were assessed by administering Depression, Anxiety and Stress Scale (DASS). T-test has been used to get the results and it is said to be statistically significant for statistical analysis and is presented in the form of tables.

Table 1

Depression among the parents of the children with life-threatening Illnesses (LTI) and Developmental Disabilities (DD).

\begin{tabular}{llllll}
\hline Group & Mean & S.D & df & t-test & $p$ \\
\hline LTI $(\mathrm{N}=50)$ & 17.42 & 5.46 & 98 & 10.21 & 0.000 \\
DD $(\mathrm{N}=50)$ & 11.93 & 5.94 & & 10.21 & 0.000 \\
\hline
\end{tabular}


Results indicate that the parents of the children with life-threatening illnesses (LTI) had higher level of depression than the parents of the children with developmental disabilities (DD).

Table 2

Anxiety among the parents of the children with life-threatening Illnesses (LTI) and Developmental Disabilities (DD)

\begin{tabular}{llllll}
\hline Group & Mean & S.D & df & t-test & $p$ \\
\hline LTI $(\mathrm{N}=50)$ & 17.54 & 5.81 & 98 & 9.77 & 0.000 \\
DD $(\mathrm{N}=50)$ & 6.20 & 5.77 & & 9.77 & 0.000 \\
\hline
\end{tabular}

Results indicate that the parents of the children with life-threatening illnesses (LTI) had higher level of anxiety than the parents of the children with developmental disabilities (DD).

Table 3

Stress among the parents of the children with life-threatening Illnesses (LTI) and Developmental Disabilities (DD)

\begin{tabular}{llllll}
\hline Group & Mean & S.D & Df & t-test & $p$ \\
\hline LTI $(\mathrm{N}=50)$ & 20.88 & 5.61 & 98 & 10.36 & 0.000 \\
DD $(\mathrm{N}=50)$ & 17.12 & 7.58 & & 10.36 & 0.000 \\
\hline
\end{tabular}

Results indicate that the parents of the children with life-threatening illnesses (LTI) had higher level of stress than the parents of the children with developmental disabilities (DD).

\section{Discussion}

This research was conducted to assess the emotional problems among the parents of the children with life-threatening illnesses and developmental disabilities. Parents share a unique bond with their children as they perceive them as their own reflection and associate their hopes, dreams and their future with them, but if their child gets a life-threatening illness or some developmental disability then all their dreams and hopes may shatter which may cause them to have emotional problems like depression, stress and anxiety. This research is however a step to assess difference in level of emotional problems in the parents of the children with lifethreatening illnesses and developmental disabilities. For this purpose 100 parents were recruited as the research participants from Allied Hospital Faisalabad, Al-Haj Siddique Aslam Sheikh Hospital Faisalabad and Tanzeem-Ul-Lisan Faisalabad. Out of those 100 parents 50 were the parents ( 25 mothers and 25 fathers) of the children with life-threatening illnesses and rest of the 50 parents ( 25 mothers and 25 fathers) were the parents of the children with developmental disabilities.

In order to assess emotional problems, Translated Urdu version of the Depression, Anxiety and Stress scale (DASS) by Aslam and Tariq (2007), was administered on the sample. According to the results of this study there is significant 
difference between the level of emotional problems among the parents of the children with life-threatening illnesses and parents of the children with developmental disabilities.

Hypothesis no.1 was "There would be a significant difference in level of depression among the parents of the children with life-threatening illnesses and the parents of the children with developmental disabilities."

This hypothesis is supported by the result and is highly significant at $\mathrm{P}<0.01$ level. It is quite clear by the Table No. 1 that the parents of the children with lifethreatening illnesses have higher level of depression than the parents of the children with developmental disabilities, because parents of the children with life-threatening illnesses are in the state of constant emergency that they have to take their child from one doctor to another doctor in order to get their child treated and to save his/her life from that fatal illness. All those lengthy hospitalizations and the suffering of their sick child and most of the times hopelessness shown by the doctors about the cure of the child's disease can cause parents to lose hope about their child's life which may cause depression in them. On the other side, parents of the children with developmental disabilities do not have to face such constant emergency as faced by the parents of the children with life-threatening-illnesses because they do not have such threat to the life of their children, this could be the reason for parents of the children with life-threatening illnesses for having high scores of depression on DASS in this study than the parents of the children with developmental disabilities.

Hypothesis no.2 was "There would be a significant difference in level of anxiety among the parents of the children with life-threatening illnesses and the parents of the children with developmental disabilities."This hypothesis is supported by the result and is highly significant at $\mathrm{P}<0.01$ level. It is quite clear by the Table No. 2 that the parents of the children with life-threatening illness have higher level of anxiety than the parents of the children with developmental disabilities. It seems that parents of the children with life-threatening illnesses have insecurities about the future of their sick child as they witness their child in pain and misery and usually are unsure about the prognosis of the disease accompanied by the fear of death of their sick child.

The literature review addressed the mental health of parents whose children have life threatening diseases and the points show that mothers have poor mental health condition in comparison to healthy mind children's' mothers (Wallander, Varni, Babani, TweddleBanis\&Wilcox, 1998). This could be the reason for parents of the children with life-threatening illnesses for having high scores of anxiety on DASS in this study than the parents of the children with developmental disabilities.

Hypothesis no. 3 was "There would be a significant difference in level of stress among the parents of the children with life-threatening illnesses and the parents of the children with developmental disabilities." This hypothesis is supported by the result and is also highly significant at $\mathrm{P}<0.01$ level. It is quite clear 
by the Table No. 3 that the parents with life-threatening illness have higher level of stress than the parents of the children with developmental disabilities. The reasons for such result could be that the parents are the primary care-givers of the children with life-threatening illnesses, so a lot of responsibilities come upon the shoulders of the parents which they try to deal with but most often they find it difficult or almost impossible to manage each and every thing at a same time which increasestheir burden and ultimately it increases their stress level.

As couple of researches also support the findings of this study that if there were uncertain outcomes of illness and intensive treatment in emergency settings,itwould increase the family stress factors and will affect the functioning of the family (Eiser, 1993; Sloper, 2000). As family functioning prior to the diagnosis of life-threatening illness is compromised, so socioeconomic hardships, disturbance in family, marital and personal life may impose great burden on the parents of the children with life-threatening illness. The maternal stress would increase if there are illness severity, PICU stay long and length of mechanical ventilation (Graves \&Ware,1990;Rivara, 1994;Youngblut\&Shiao, 1993).

Contrary to that the parents of the children with developmental disabilities do not have such emergency and uncertain conditions about their child's life. Moreover the family life of the parents of the children with developmental disabilities do not get as much disturbed as that of the parents of the children with life-threatening illnesses because they do not have to face frequent hospitalizations and they also do not have such threat of death of their child as the parents of the children with life-threatening illness have, so this may be the reason for the parents of the children with life-threatening illness for having high scores of stress on DASS in this research than the parents of the children with developmental disabilities.

\section{Conclusion}

The current research was designed to assess the emotional problems in the parents whose children have life threatening disease and developmental disabilities. A sample of 100 parents was selected from different Government and private hospitals of Faisalabad and school of those children who have special needs. The sample is divided into $n=50$ parents whose children have any life threatening disease and $n=50$ parents of children who have developmental disabilities. Translated Urdu version of the questionnaire has been developed by Aslam and Tariq (2007) to measure the emotional problems. $T$ test has been used for the statistical analysis. The results have shown the significance difference in the levels of emotional problems among parents of children who have life-threatening illnesses and developmental disabilities. On the basis of current results, it is recommended that in future studies gender difference may also be compared. 


\section{References}

Aslam, N., \& Tariq, N. (2010). Trauma, depression, anxiety, and stress among individuals living in earthquake affected and unaffected areas. Pakistan Journal of Psychological Research, 131-148.

Benini, F., Spizzichino, M., Trapanotto, M., \& Ferrante, A. (2008). Pediatric palliative care.Italian Journal of Pediatrics, 34-4.

Cadman, D., Rosenbaum, P., Boyle, M., \& Oxford, D.R. (1991). Children with chronicillness: Family and parent demographic characteristics and psychological adjustment. Pediatrics, 87, 884-889.

Cohen, M.S. (1999). Families coping with childhood chronic illness: A research review. Families, Systems, and Health, 17, 149-164.

Daoud, M. S, Dooley, J. M., \& Gordon, K. E. (2004). Depression in parents of children with Duchenne muscular dystrophy.Pediatric Neurology, 31, 16-19.

Dunn, M. E., Burbine, T., Bowers, C. A., \&Tantleff-Dunn, S. (2001). Moderators of stress in parents of children with autism. Community Mental Health Journal, 37, 39-52.

Eiser, C. (1993). Growing up with a chronic disease: The impact on children and families. London: Jessica Kingsley Publishers.

Feldman, L. A. (1993). The role of Positive affect in discriminating self-reported depression from anxiety. Journal of health and Social behavior, 7, 125-135.

Graves, J. K., \& Ware, M. E. (1990). Parents' and health professionals' perceptions concerning parental stress during a child's hospitalization. Child Health Care, 19, $37-42$.

Hastings, R.P., Kovshoff, H., Brown, T., Ward, N. J., Espinosa, F. D., \& Remington, B. (2005). Coping strategies in mothers and fathers of preschool and school-age children with autism. Autism, 9, 377-391.

Lovibond, S. H., \&Lovibond, P.F. (1995). Manual for the depression anxiety and stress scale. Sydney: Psychology foundation.

Olsson, M. B., \& Hwang, C. P. (2001). Depression in mothers and fathers of children with intellectual disability. Journal of Intellectual Disability Research, 45, 535-543.

Robins, S. L., \&Cotran, R. S. (2004). Pathalogical Basis of Diseases. New Delhi: Elsevier.

Sloper, P. (2000). Predictors of distress in parents of children with cancer: A prospective study. Journal of Pediatric Psychology, 25, 79-91. 
Steele, R., \&Davies, B. (2006). Impact on parents when a child has a progressive, lifethreatening illness. Journal of Palliative Nursing, 12, 576-85.

Steinglass, P. (1998). Multiple family discussion groups for patients with chronic medical illness. Families, Systems, and Health, 16, 55-70.

Tiedje, LB., \& Darling-Fisher, C.S. (1996). Fatherhood reconsidered: A critical review. Research in Nursing and Health, 19, 471-484.

Vitaliano, P. P., Zhang, J., \&Scanlan, J. M. (2003). Is caregiving hazardous to one's physical health? A meta-analysis.Psychology Bulletin, 129, 946-72.

Wallander, J.L., Varni, J.W., Babani, L., TweddleBanis, H., \& Wilcox, T. (1989). Family resources as resistance factors for psychological mal-adjustment in chronically ill and handicapped children. Journal of Pediatric Psychology, 14, 157173.

Yirmiya, N., \&Shaked, M. (2005). Psychiatric disorders in parents of children with autism: A meta-analysis. Journal of Child Psychology E Psychiatry, 46, 69-83.

Youngblut, J. M., \&Shiao, S. P. (1993). Child and family reactions during and after pediatric ICU hospitalization: a pilot study. Heart Lung, 22, 46-54. 